\title{
Basal contralateral aldosterone suppression is rare in lateralized primary aldosteronism
}

\section{Marie-Josée Desrochers', Matthieu St-Jean', Nada El Ghorayeb', Isabelle Bourdeau1, Benny So ${ }^{4}$, Éric Therasse ${ }^{2}$, Gregory Kline ${ }^{3}$ and André Lacroix (D) 1}

${ }^{1}$ Division of Endocrinology, Department of Medicine, ${ }^{2}$ Department of Radiology, Centre de Recherche du Centre hospitalier de I'Université de Montréal (CHUM), Université de Montréal, Québec, Canada, ${ }^{3}$ Division of Endocrinology, Department of Medicine, and ${ }^{4}$ Department of Radiology, University of Calgary, Alberta, Canada
Correspondence

should be addressed

to A Lacroix

Email

andre.lacroix@umontreal.ca

\begin{abstract}
Context: Unilateral aldosteronomas should suppress renin and contralateral aldosterone secretion. Complete aldosterone suppression in contralateral adrenal vein sample (AVS) could predict surgical outcomes. Objectives: To retrospectively evaluate the prevalence of basal contralateral suppression using Aldosterone $(\mathrm{A})_{\text {contralateral(CL) }} / \mathrm{A}_{\text {peripheral(P) }}$ as compared to $\left(\mathrm{A} / \mathrm{Cortisol}(\mathrm{C})_{\mathrm{CL}}\right) /(\mathrm{A} / \mathrm{C})_{\mathrm{P}}$ ratio in primary aldosteronism (PA) patients studied in two Canadian centers. To determine the best cut-off to predict clinical and biochemical surgical cure. To compare the accuracy of $A_{C L} / A_{P}$ to the basal and post-ACTH lateralization index (LI) in predicting surgical cure.

Methods: In total, 330 patients with PA and successful AVS were included; 124 lateralizing patients underwent surgery. Clinical and biochemical cure at 3 and 12 months were evaluated using the PASO criteria.

Results: Using $A_{C L} / A_{P}$ and $(A / C)_{C L} /(A / C)_{P}$ at the cut-off of 1, the prevalence of contralateral suppression was 6 and $45 \%$, respectively. Using ROC curves, the $A_{C L} / A_{p}$ ratio is associated with clinical cure at 3 and 12 months and biochemical cure at 12 months. $(A / C)_{C L} /(A / C)_{P}$ is associated with biochemical cure only. The cut-offs for $A_{C L} / A_{p}$ offering the best sensitivity (Se) and specificity (Sp) for clinical and biochemical cures at 12 months are 2.15 (Se: 63\% and Sp: 71\%) and 6.15 (Se: 84\% and Sp: 77\%), respectively. Basal LI and post-ACTH LI are associated with clinical cure but only the postACTH LI is associated with biochemical cure.

Conclusions: In lateralized PA, basal contralateral suppression defined by $\mathrm{A}_{\mathrm{CL}} / \mathrm{A}_{\mathrm{P}}$ is rare and incomplete compared to the $(A / C)_{C L} /(A / C)_{P}$ ratio and is associated with clinical and biochemical postoperative outcome, but with modest accuracy.
\end{abstract}

\section{Introduction}

Primary aldosteronism (PA) affects approximately 5\% of hypertensive patients in the general population, up to $20 \%$ with resistant hypertension and is associated with increased morbidity and mortality compared to essential hypertension $(1,2,3,4)$. The two major subtypes of PA are bilateral adrenal hyperplasia $(\mathrm{BAH})$ and unilateral aldosterone-producing adenoma (APA). In confirmed PA cases, the source of aldosterone excess should be identified to guide the treatment and reverse the complications associated with the disease (5). The most appropriate treatment remains unilateral adrenalectomy for APA and effective mineralocorticoid receptor blockade for BAH. Adrenal vein sampling (AVS) remains the procedure of choice to differentiate lateralized from bilateral source of aldosterone (5). However, there is great discrepancy in the protocols and interpretation of AVS without standardization between referral centers $(6,7)$.

A suppression of the contralateral aldosterone production should be found in cases of excess aldosterone secretion by a unilateral APA as sodium retention and 
blood volume expansion suppresses the renin-angiotensin system. In several publications, contralateral aldosterone suppression was measured in AVS following ACTH administration; in most studies, it is calculated using the cortisol-corrected aldosterone ratio in contralateral adrenal vein/peripheral vein $(8,9,10,11,12,13)$. It was reported that, in patients with lateralized aldosterone source, contralateral suppression using cortisol-corrected aldosterone ratio $(\leq 1)$ was frequent $(8,9,10,11,12,13)$. However, postoperative hypoaldosteronism associated with hypotension and hyperkaliemia is present in less than $5 \%$ of patients after unilateral adrenalectomy for APA, despite contralateral adrenal suppression calculated with these criteria $(14,15)$. This suggests that contralateral suppression based on cortisol-corrected and post-ACTH ratio may be inaccurate to evaluate aldosterone secretion of the non-dominant adrenal. Pathology studies showed frequently zona glomerulosa hyperplasia and nodulation adjacent to APA that lateralized during AVS, which suggests that a certain degree of zona glomerulosa hyperplasia is present in the contralateral adrenal (16, 17). Thus, a lack of complete contralateral aldosterone suppression could be a useful marker to assess the presence of contralateral zona glomerulosa nodular hyperplasia and help to predict postoperative outcome, but this remains controversial $(8,9,10,11,12)$. We recently found that contralateral aldosterone suppression was relatively rare using the ratio of basal aldosterone concentration of the contralateral adrenal vein/periphery $\left(\mathrm{A}_{\mathrm{CL}} / \mathrm{A}_{\mathrm{P}}\right)$ in contrast to the traditional cortisol-corrected aldosterone ratio $\left((\mathrm{A} / \mathrm{C})_{\mathrm{CL}}(\mathrm{A} / \mathrm{C})_{\mathrm{P}}\right)(14)$. We questioned the purpose of correcting the aldosterone concentration by cortisol in peripheral vein as their secretion mechanisms are distinct. There is no reason to correct aldosterone by cortisol in the peripheral vein as there is no correction requirement for blood flow or dilution phenomenon. We also questioned the rationale of calculating this ratio after stimulation with ACTH, as the objective is to evaluate the degree of suppression of aldosterone secretion by the contralateral adrenal. We previously reported in a small subset of surgically treated APA $(n=66)$ that the absence of basal contralateral suppression using $A_{C L} / A_{P}$ was associated with a lower rate of clinical and biochemical improvement. In contrast, the absence of basal contralateral suppression using $(\mathrm{A} / \mathrm{C})_{\mathrm{CL}}(\mathrm{A} / \mathrm{C})_{\mathrm{P}}$ was not predictive of a lower response to unilateral adrenalectomy in the same subset of patients (14). In this study, we have examined the performance of contralateral aldosterone suppression ratio using basal $A_{C L} / A_{P}$ ratio compared to $(\mathrm{A} / \mathrm{C})_{\mathrm{CL}}(\mathrm{A} / \mathrm{C})_{\mathrm{P}}$ ratio and to the $\mathrm{LI}$ ratio in a larger cohort of patients with PA in two Canadian centers that use the same simultaneous bilateral AVS technique with basal and post-ACTH samples.

\section{Subjects and methods}

\section{Patients}

We retrospectively reviewed the medical records of 192 consecutive patients in the Montreal cohort (including 92 previously reported patients) (14) and 138 patients in the Calgary cohort who underwent AVS between 2009 and 2018 and 2005 and 2018, respectively (see Flowchart in Supplementary materials, see section on supplementary materials given at the end of this article). Patients gave written informed consent to perform the AVS. Institutional review board at the CHUM (Comité d'éthique du Centre hospitalier de l'Université de Montréal) waived requirement to obtain written consent from the patients to analyze and publish theses results and granted permission for the retrospective and anonymous analysis and publication of the data. The Conjoint Health Research Ethics Board at the Calgary University requested patients to give written informed consent to collect patient's data from Foothills Medical Center.

Demographic data including patient age, sex and $\mathrm{BP}$ were recorded, as well as the use of antihypertensive medications, potassium supplementation, results of biochemical and imaging studies. PA diagnosis was established according to the current Endocrine Society guidelines and long-standing published local approaches to the definition of PA $(5,8) .1 \mathrm{mg}$ overnight dexamethasone suppression test was conducted in every patient before AVS to evaluate for potential cortisol cosecretion and interference with AVS interpretation.

\section{Adrenal venous sampling procedure, selectivity and lateralization index and hormone assays}

Described in Supplementary data.

\section{Definition of basal contralateral aldosterone suppression ratio}

Contralateral suppression was calculated based on basal values in two different manners:

1) Basal contralateral suppression ratio=mean of $(\mathrm{A} / \mathrm{C})_{\mathrm{CL}} /(\mathrm{A} / \mathrm{C})_{\mathrm{P}}$ at $\mathrm{t}-5$ and $\mathrm{t} 0$ for the Montreal cohort and $(\mathrm{A} / \mathrm{C})_{\mathrm{CL}} /(\mathrm{A} / \mathrm{C})_{\mathrm{P}}$ at $\mathrm{t} 0$ for the Calgary cohort. 
2) Basal contralateral suppression ratio $=$ mean of $A_{C L} / A_{P}$ at $\mathrm{t}-5$ and $\mathrm{t} 0$ for the Montreal cohort and $\mathrm{A}_{\mathrm{CL}} / \mathrm{A}_{\mathrm{P}}$ at to for the Calgary cohort.

\section{Data analysis and criteria for adrenalectomy}

AVS were considered eligible for inclusion if they had selectivity index (SI) greater than 5 after ACTH stimulation bilaterally. Among these bilaterally selective AVS, patients with a basal lateralization index (LI) greater than 2 and a post-ACTH LI greater than 4 in Montreal and an LI greater than 3 after ACTH in Calgary were considered as having lateralized disease and offered unilateral adrenalectomy. Some patients not completely meeting these criteria were operated based on the presence of contralateral suppression with elevated basal LI only (Montreal group) and on the treating physician judgment and therefore were included in the analysis.

\section{Pre-established cut-offs of contralateral aldosterone suppression}

Contralateral aldosterone suppression ratio was evaluated at different pre-established cut-offs that have been used in the literature or found to be predictive of postoperative cure in a previous study published by the Montreal group (14). We, therefore, evaluated the two different ratios at the traditional cut-off of 1 and also at the cut-off of 1.5 for $\mathrm{A}_{\mathrm{CL}} / \mathrm{A}_{\mathrm{P}}$ and 0.66 for $(\mathrm{A} / \mathrm{C})_{\mathrm{CL}} /(\mathrm{A} / \mathrm{C})_{\mathrm{P}}$.

\section{Evaluation of outcome following unilateral adrenalectomy}

A total of 124 patients underwent unilateral adrenalectomy, 72 patients from the Montreal cohort and 52 patients from the Calgary cohort. Clinical and biochemical outcomes were evaluated at 3 months and also at the last follow-up after a minimum of 12 months. In the surgical group, patients with missing data or with less than 3 months of follow-up were also excluded (see Flowchart in Supplementary materials). The criteria used to define clinical and biochemical improvement were the most recently established criteria by the PASO international consensus (18). See Supplementary data for full description.

\section{Statistical analysis}

Continuous variables are presented as means \pm S.D. or median (IQR) and categorical variables as counts and percentages. Patient's baseline clinical characteristics between Montreal and Calgary cohorts were compared using chi-squared test for categorical variables and Kruskal-Wallis test or t-test for continuous variables, depending on the parametric fit of the data. Receiver operating characteristic (ROC) analysis were performed separately for four predictors $\left(\mathrm{A}_{\mathrm{CL}} / \mathrm{A}_{\mathrm{P}},(\mathrm{A} / \mathrm{C})_{\mathrm{CL}} /(\mathrm{A} / \mathrm{C})_{\mathrm{P}}\right.$, basal LI and post-ACTH LI) and for two outcomes (clinical and biochemical cure of PA for patients who underwent unilateral adrenalectomy) at 3 and 12 months in combined Montreal and Calgary cohorts. The binary clinical and biochemical cure outcomes were defined as complete vs combined partial and absent cure. The AUC with 95\% CI and the differences of AUC's with 95\% CI were calculated to evaluate the statistical significance of the predictors (19) and to compare these predictors (20). The optimal cutpoint values were obtained using the point closest-to- $(0,1)$ approach (21). The respective sensitivities and specificities were reported. The distributions of contralateral suppression, ROC curves and the AUC results were also presented in the graphical forms. All tests were conducted at two-sided 0.05 level of significance. Statistical software SAS version 9.4 was used for all calculations (SAS Institute, Cary, NC, USA).

\section{Results}

\section{Clinical features}

The clinical, biochemical and radiological parameters of the patients included in the study are shown in Table 1. In both cohorts $(n=330)$, the mean age was $52 \pm 12$ years and $63 \%$ were males. Mean BP under antihypertensive medication at the initial visit was 144 (130-156)/90 (80-96) mmHg; the Calgary cohort presented a higher diastolic blood pressure (DBP) than the Montreal cohort $(P=0.006)$. The potassium levels were also lower in the Calgary cohort $(3.2(2.8-3.7) \mathrm{mmol} / \mathrm{L})$ compared to the Montreal cohort (3.6 (3.3-4.0) pmol/L) $(P<0.0001)$. The plasma aldosterone concentration (PAC) and plasma renin activity (PRA) were statistically different between cohorts, but the ARR was comparable. The basal and postACTH LI were higher in the Montreal cohort compared to the Calgary cohort. Patients in the Montreal cohort were treated with more antihypertensive drugs compared to Calgary $(P<0.0001)$. In the surgical cohort, data were comparable between the Montreal and Calgary cohorts, with the exception of the potassium level and a number of antihypertensive medications. 


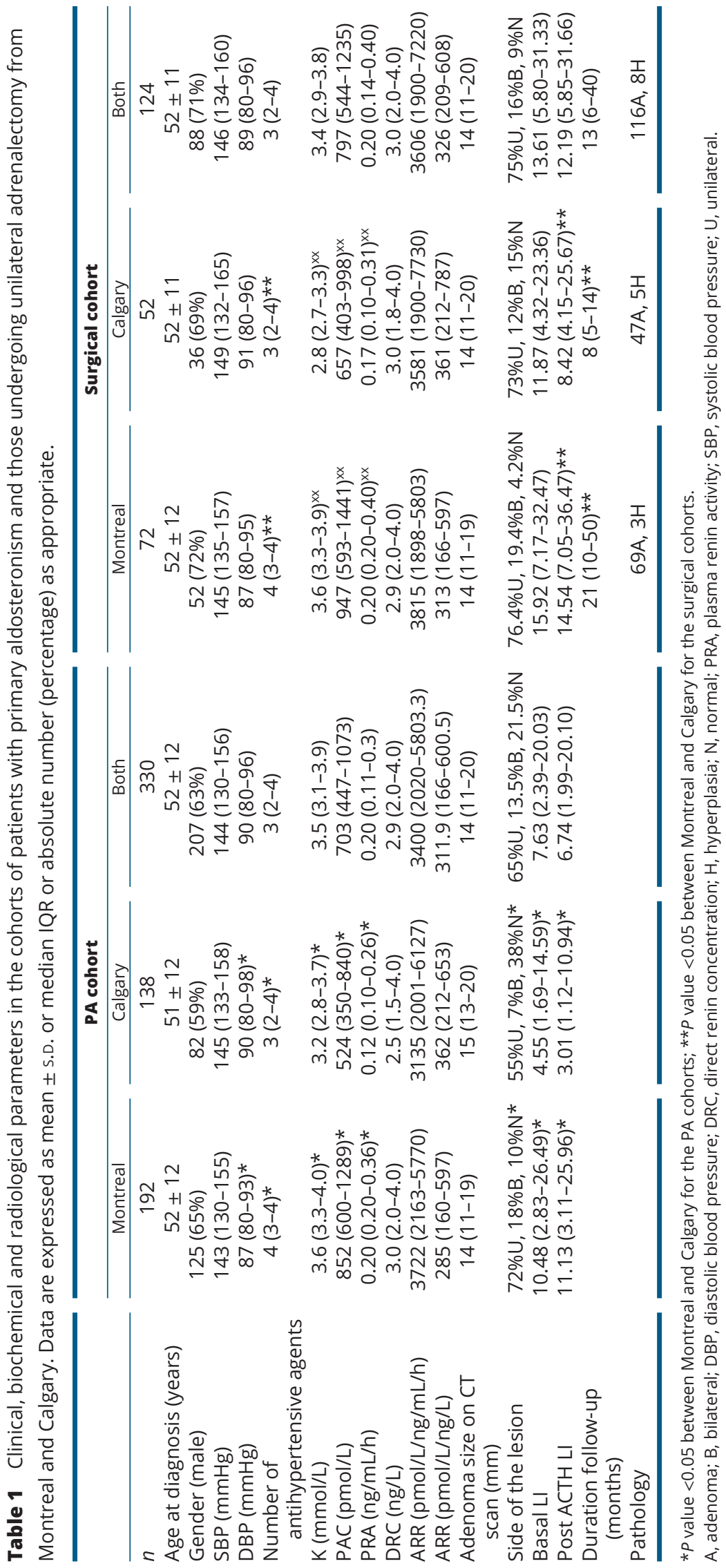




\section{Evaluation of basal contralateral aldosterone suppression}

In both cohorts with a total of 330 PA cases, the prevalence of contralateral suppression at the traditional cut-off of 1 was present in $45 \%$ of patients using the $(\mathrm{A} / \mathrm{C})_{\mathrm{CL}} /$ $(\mathrm{A} / \mathrm{C})_{\mathrm{P}}$ ratio but only in $6 \%$ of patients using the $\mathrm{A}_{\mathrm{CL}} / \mathrm{A}_{\mathrm{P}}$ ratio (Fig. 1 and Table 2). The difference was statistically significant between the Montreal and the Calgary cohort for the prevalence of contralateral suppression using the $(\mathrm{A} / \mathrm{C})_{\mathrm{CL}} /(\mathrm{A} / \mathrm{C})_{\mathrm{P}}$ ratio, being more frequent in patients from Montreal. Of note, in peripheral samples, the PAC was statistically higher (418 vs $354 \mathrm{pmol} / \mathrm{L} ; P=0.034)$ and the cortisol was statistically lower (216 vs $280 \mathrm{nmol} / \mathrm{L}$; $P<0.0001)$ in the Montreal cohort compared to the Calgary cohort. PAC and cortisol in the contralateral adrenal vein were similar between groups, which results in a higher denominator for the $(\mathrm{A} / \mathrm{C})_{\mathrm{CL}} /(\mathrm{A} / \mathrm{C})_{\mathrm{P}}$ ratio and potentially a higher proportion of patients from Montreal with contralateral suppression in these conditions (data not shown). The different cut-offs for adrenalectomy and different immunoassay used between centers could explain this variation. Among the 219 lateralized cases of $\mathrm{PA}$, contralateral aldosterone suppression at the traditional cut-off of 1 was present in $56 \%$ of patients of the total cohorts using the $(\mathrm{A} / \mathrm{C})_{\mathrm{CL}} /(\mathrm{A} / \mathrm{C})_{\mathrm{P}}$ ratio and in only $7 \%$ of patients using the $A_{C L} / A_{P}$ ratio (Table 2).

The median contralateral aldosterone suppression ratio is $2.3(1.3-5.1)$ for the lateralized cases and 14.0 (6.0-

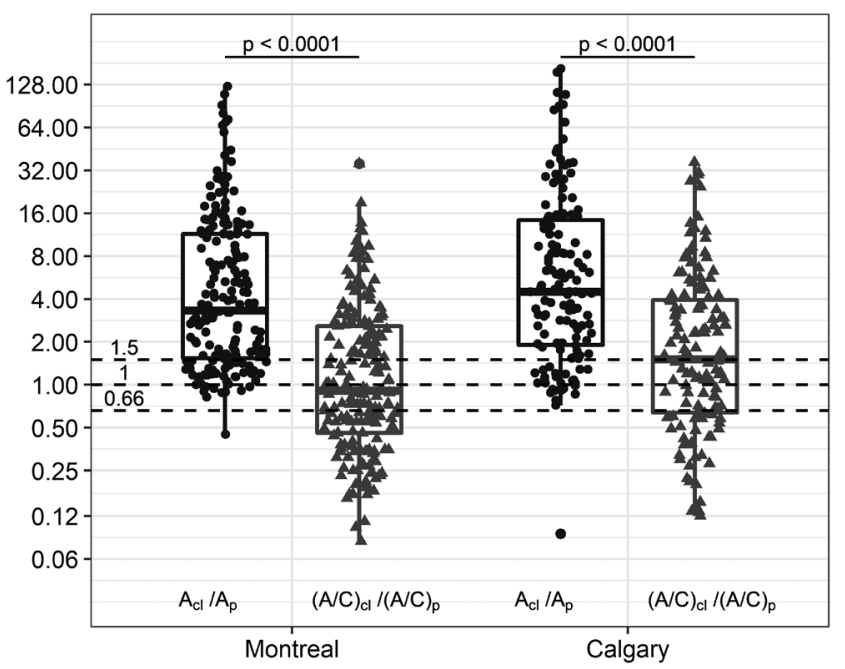

\section{Figure 1}

Comparison of the proportion of contralateral suppression in the cohorts of patients with PA from Montreal $(n=192)$ and Calgary ( $n=138$ ) using two different methods to calculate the ratios.
27.6) for the bilateral disease using $A_{C L} / A_{P}$ compared to 0.7 $(0.4-1.3)$ and $3.6(2.1-7.6)$ for $(\mathrm{A} / \mathrm{C})_{\mathrm{CL}} /(\mathrm{A} / \mathrm{C})_{\mathrm{P}}$, respectively. There was no statistically significant difference between the Montreal and Calgary cohorts.

\section{Evaluation of clinical and biochemical cures after unilateral adrenalectomy}

The median follow-up after unilateral adrenalectomy was 21 (10-50) months for the Montreal cohort and 8 (5-14) months for the Calgary cohort (Table 1). Adequate data to determine the postoperative cures at 3 months were available in $98 \%$ for the clinical cure and $90 \%$ for the biochemical cure for the combined surgical cohorts. Fewer data were available at 12 months: 57 patients (79\%) and 56 patients $(78 \%)$ for clinical and biochemical cures in the Montreal cohort, respectively, and 25 patients (48\%) and 21 patients (40\%) for clinical and biochemical cures in the Calgary cohort, respectively. At 12 months, complete and partial clinical cures were $29 \%$ and $67 \%$, respectively; results were similar at 3 months and between cohorts. In total, $83 \%$ of the cohort presented complete biochemical cure at 12 months and the results were similar at 3 months. At 12 months, $27 \%$ of patients presented both complete clinical and biochemical success (34\% for Montreal and $10 \%$ for Calgary) (Table 3).

\section{Evaluation of the postoperative parameters according to basal contralateral aldosterone suppression}

Since there is no consensus for the cut-off of contralateral suppression, we conducted a ROC analysis using contralateral suppression results as a predictor of complete clinical cure compared to partial and absent clinical cure and performed the same analysis for the biochemical cure (Fig. 2). The $A_{C L} / A_{p}$ ratio is associated with clinical cure at 3 and at 12 months (AUC 0.71 (95\% CI: 0.61-0.81) and 0.72 (95\% CI: $0.60-0.84)$ ), while the $(\mathrm{A} / \mathrm{C})_{\mathrm{CL}} /(\mathrm{A} / \mathrm{C})_{\mathrm{P}}$ is not associated (AUC 0.60 (95\% CI: 0.50-0.70) and 0.55 (95\% CI: $0.42-0.68))(P=0.01$ and $P=0.037)$. The AUC's of $(\mathrm{A} / \mathrm{C})_{\mathrm{CL}} /$ $(\mathrm{A} / \mathrm{C})_{\mathrm{P}}$ are significantly smaller comparing with $\mathrm{A}_{\mathrm{CL}} / \mathrm{A}_{\mathrm{P}}$ ratio for the clinical cure at 3 and at 12 months $(P=0.037$ and $P=0.01$ respectively) (Table 4 ). The best combination of specificity and sensitivity for the clinical cure at 12 months for $\mathrm{A}_{\mathrm{CL}} / \mathrm{A}_{\mathrm{P}}$ predictor was obtained at a ratio of 2.15 (Sp, 71\%; Se, 63\%), using the point closest-to-(0.1) technique. For the evaluation of the biochemical cure at 12 months, the two ratios were associated with the postoperative outcome (AUC 0.83 (95\% CI: 0.72-0.94) 
Table 2 Percentage of patients presenting contralateral aldosterone suppression in AVS depending on the method used to calculate the ratio and the cut-offs used. Data are expressed in absolute number (percentage).

\begin{tabular}{|c|c|c|c|c|}
\hline Cut-off & Montreal & Calgary & Both & $P$ value* \\
\hline \multicolumn{5}{|l|}{ Total cohort } \\
\hline Number of patients & 192 & 138 & 330 & \\
\hline \multicolumn{5}{|l|}{$A_{C L} / A_{P}$} \\
\hline 1.0 & $9(5 \%)$ & $11(8 \%)$ & $20(6 \%)$ & 0.318 \\
\hline 1.5 & $47(25 \%)$ & $26(19 \%)$ & $73(22 \%)$ & 0.279 \\
\hline \multicolumn{5}{|l|}{$(\mathrm{A} / \mathrm{C})_{\mathrm{CL}} /(\mathrm{A} / \mathrm{C})_{\mathrm{P}}$} \\
\hline 1.0 & $100(52 \%)$ & $49(36 \%)$ & $149(45 \%)$ & 0.004 \\
\hline 0.66 & $75(39 \%)$ & $35(25 \%)$ & $110(33 \%)$ & 0.013 \\
\hline \multicolumn{5}{|l|}{ Lateralized source } \\
\hline Number of patients & 141 & 78 & 219 & \\
\hline \multicolumn{5}{|l|}{$\mathrm{A}_{\mathrm{CL}} / \mathrm{A}_{\mathrm{p}}$} \\
\hline 1.0 & $9(6 \%)$ & $10(13 \%)$ & $19(9 \%)$ & 0.105 \\
\hline 1.5 & $47(33 \%)$ & $25(32 \%)$ & $72(33 \%)$ & 0.847 \\
\hline \multicolumn{5}{|l|}{$(\mathrm{A} / \mathrm{C})_{\mathrm{CL}} /(\mathrm{A} / \mathrm{C})_{\mathrm{P}}$} \\
\hline 1.0 & $98(70 \%)$ & $46(59 \%)$ & $144(66 \%)$ & 0.116 \\
\hline 0.66 & $74(53 \%)$ & $33(42 \%)$ & $107(49 \%)$ & 0.149 \\
\hline \multicolumn{5}{|l|}{ Bilateral source } \\
\hline Number of patients & 51 & 60 & 111 & \\
\hline \multicolumn{5}{|l|}{$\mathrm{A}_{\mathrm{CL}} / \mathrm{A}_{\mathrm{P}}$} \\
\hline 1.0 & $0(0 \%)$ & $1(2 \%)$ & $1(1 \%)$ & 0.354 \\
\hline 1.5 & $0(0 \%)$ & $1(2 \%)$ & $1(1 \%)$ & 0.354 \\
\hline \multicolumn{5}{|l|}{$(\mathrm{A} / \mathrm{C})_{\mathrm{CL}} /(\mathrm{A} / \mathrm{C})_{\mathrm{P}}$} \\
\hline 1.0 & $2(4 \%)$ & $3(5 \%)$ & $5(5 \%)$ & 0.785 \\
\hline 0.66 & $1(2 \%)$ & $2(3 \%)$ & $3(3 \%)$ & 0.657 \\
\hline
\end{tabular}

The cut-off used to calculate the percentage of patient with contralateral aldosterone suppression are presented in boldface.

${ }^{*}$ Chi-squared or exact Fisher test to compare Montreal and Calgary cohorts.

Table 3 Classification and prevalence of the postoperative clinical and biochemical outcomes at 3 and 12 months in the surgically treated PA patients. Data are expressed in absolute number (percentage).

\begin{tabular}{|c|c|c|c|}
\hline Postoperative outcome & Montreal $(n=72)$ & Calgary $(n=52)$ & Both $(n=124)$ \\
\hline \multicolumn{4}{|l|}{3 months } \\
\hline \multicolumn{4}{|l|}{ Clinical success } \\
\hline Complete & $23(33 \%)$ & $14(27 \%)$ & $37(30 \%)$ \\
\hline Partial & $46(66 \%)$ & $35(67 \%)$ & $81(67 \%)$ \\
\hline Absent & $1(1 \%)$ & $3(6 \%)$ & $4(3 \%)$ \\
\hline \multicolumn{4}{|l|}{ Biochemical success } \\
\hline Complete & $58(87 \%)$ & $36(82 \%)$ & $94(85 \%)$ \\
\hline Partial & $2(3 \%)$ & $5(11 \%)$ & $7(6 \%)$ \\
\hline Absent & $7(10 \%)$ & $3(7 \%)$ & $10(9 \%)$ \\
\hline Both clinical and biochemical complete success & $21(31 \%)$ & $9(20 \%)$ & $30(27 \%)$ \\
\hline \multicolumn{4}{|l|}{12 months } \\
\hline \multicolumn{4}{|l|}{ Clinical success } \\
\hline Complete & $20(35 \%)$ & $4(16 \%)$ & $24(29 \%)$ \\
\hline Partial & $36(63 \%)$ & $19(76 \%)$ & $55(67 \%)$ \\
\hline Absent & $1(2 \%)$ & $2(8 \%)$ & $3(4 \%)$ \\
\hline \multicolumn{4}{|l|}{ Biochemical success } \\
\hline Complete & $47(84 \%)$ & $17(81 \%)$ & $64(83 \%)$ \\
\hline Partial & $2(4 \%)$ & $1(5 \%)$ & $3(4 \%)$ \\
\hline Absent & $7(12 \%)$ & $3(14 \%)$ & $10(13 \%)$ \\
\hline Both clinical and biochemical complete success & $19(34 \%)$ & $2(10 \%)$ & $21(27 \%)$ \\
\hline
\end{tabular}


A
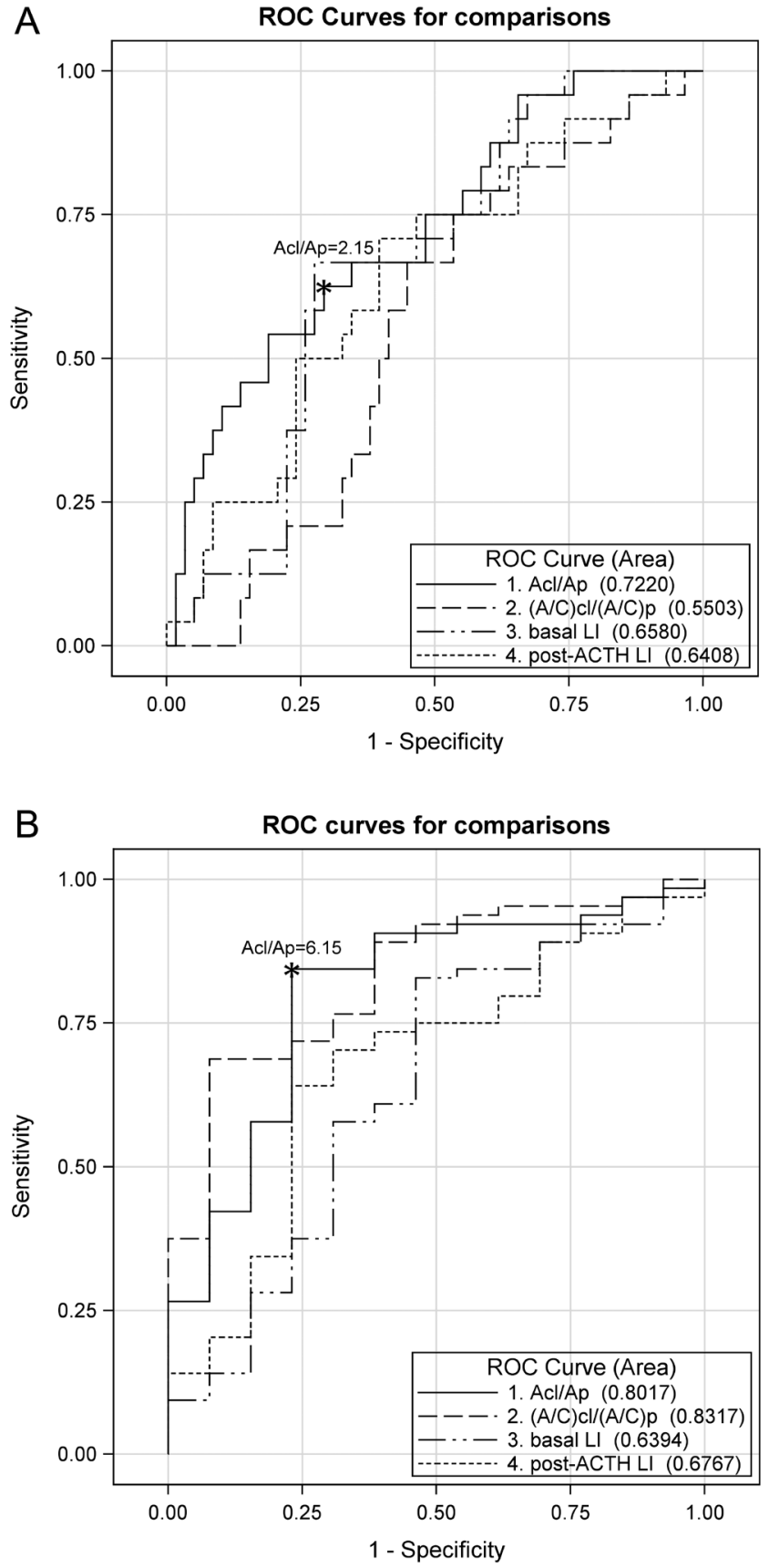

Figure 2

ROC curves for the comparisons of the four predictors at 12 months in the combined PA surgical cohorts for clinical $(A)$ and biochemical (B) cures.

for the $(\mathrm{A} / \mathrm{C})_{\mathrm{CL}} /(\mathrm{A} / \mathrm{C})_{\mathrm{P}}$ ratio and 0.80 (95\% CI: 0.66-0.94) for the $A_{C L} / A_{P}$ ratio and these AUC's were not statistically different $(P=0.545)$ ) (Table 4$)$. The best combination of specificity and sensitivity for biochemical cure using the $\mathrm{A}_{\mathrm{CL}} / \mathrm{A}_{\mathrm{P}}$ method was obtained at a ratio of 6.15 (Sp, 77\%; Se, $84 \%)$. Patients with contralateral aldosterone suppression
Table 4 Area under the curve analysis for clinical and biochemical cures at 3 and 12 months in combined Montreal and Calgary cohorts. Data are expressed in absolute number of AUC $(95 \% \mathrm{CI})$.

Outcome/predictor

AUC $(95 \% \mathrm{Cl})$ *

Clinical cure at 3 months

$A_{C L} / A_{P}$

$(\mathrm{A} / \mathrm{C})_{\mathrm{CL}} /(\mathrm{A} / \mathrm{C})_{\mathrm{P}}$

Basal LI

Post ACTH LI

Biochemical cure at 3 months

$\mathrm{A}_{\mathrm{CL}} / \mathrm{A}_{\mathrm{P}}$

$(\mathrm{A} / \mathrm{C})_{\mathrm{CL}} /(\mathrm{A} / \mathrm{C})_{\mathrm{P}}$

Basal LI

Post ACTH LI

Clinical cure at 12 months

$\mathrm{A}_{\mathrm{CL}} / \mathrm{A}_{\mathrm{P}}$

$(\mathrm{A} / \mathrm{C})_{\mathrm{CL}} /(\mathrm{A} / \mathrm{C})_{\mathrm{P}}$

Basal LI

Post ACTH LI

Biochemical cure at 12 months

$\mathrm{A}_{\mathrm{CL}} / \mathrm{A}_{\mathrm{P}}$

$(\mathrm{A} / \mathrm{C})_{\mathrm{CL}} /(\mathrm{A} / \mathrm{C})_{\mathrm{P}}$

Basal LI

Post ACTH LI

$0.71(0.61-0.81)$

$0.60(0.49-0.70)^{* *}$

$0.64(0.54-0.74)$

$0.64(0.54-0.75)$

$0.65(0.49-0.82)$

$0.72(0.59-0.85)$

$0.57(0.29-0.85)$

$0.65(0.54-0.75)$

$0.72(0.60-0.84)$

$0.55(0.42-0.68)^{* *}$

$0.66(0.54-0.78)$

$0.64(0.51-0.77)$

$0.80(0.66-0.94)$

$0.83(0.72-0.94)$

$0.64(0.46-0.82)^{* *}$

$0.68(0.51-0.84)$

*95\% $\mathrm{Cl}$ for the area under the curve (AUC) were computed using DeLong method and ${ }^{*} P$ value $<0.05$ of the difference of $A \cup C$ of $(A / C)_{O P P} /(A / C)_{P \prime}$ basal $\mathrm{LI}$ and post-ACTH LI predictors compared to $\mathrm{A}_{\mathrm{OPP}} / \mathrm{A}_{\mathrm{P}}$.

$<2.15$ had a significantly more severe form of the disease (Table 5).

\section{Comparison of the contralateral aldosterone suppression ratio $\left(A_{C L} / A_{p}\right)$ to the basal and post- ACTH lateralization ratio (LI) for the prediction of the postoperative outcome}

ROC analysis for the basal LI and the post-ACTH LI were conducted simultaneously on the combined cohorts and used the postoperative outcomes at 12 months (Fig. 2). Both LI ratios can predict the clinical outcome as shown by their AUC of 0.66 (95\% CI: 0.54-0.78) and 0.64 (95\% CI: 0.51-0.77) for the basal LI and post-ACTH LI, respectively. For the biochemical outcome, only the postACTH LI is predictive (AUC 0.68 (95\% CI: 0.51-0.84)) (Table 4). The best combination of specificity and sensitivity regarding clinical outcome were obtained at the cut-offs of 16.51 (Sp: 72\%; Se: 67\%) and 12.49 (Sp: 60\%; Se: 71\%) for basal LI and post ACTH LI, respectively, using the point closest-to-(0.1) technique. These cut-offs were 5.21 (Sp: 54\%; Se: 83\%) and 8.44 (Sp: 77\%; Se: 64\%) for the biochemical outcome using basal and post-ACTH LIs, respectively (data not shown). Patients with post 
Table 5 Clinical, biochemical and radiological parameters of PA patients according to basal contralateral suppression. Data are expressed as mean \pm S.D. or median IQR or absolute number (percentage) as appropriate.

\begin{tabular}{|c|c|c|c|}
\hline & $\mathbf{A}_{\mathbf{c L}} / \mathbf{A}_{\mathbf{p}} \leq \mathbf{2 . 1 5}(n=113)$ & $\mathbf{A}_{\mathrm{CL}} / \mathbf{A}_{\mathbf{p}}>\mathbf{2 . 1 5}(n=217)$ & P-value \\
\hline Age at diagnosis (years) & $50 \pm 13$ & $52 \pm 11$ & 0.078 \\
\hline Gender (male) & $65(58 \%)$ & $142(65 \%)$ & 0.158 \\
\hline $\mathrm{SBP}(\mathrm{mmHg})$ & $144(132-160)$ & $144(130-154)$ & 0.562 \\
\hline $\mathrm{DBP}(\mathrm{mmHg})$ & $90(80-97)$ & $89(80-96)$ & 0.982 \\
\hline Number of antihypertensive agents & $3(2-4)$ & $3(2-4)$ & 0.036 \\
\hline $\mathrm{K}(\mathrm{mmol} / \mathrm{L})$ & $3.3(2.8-3.7)$ & $3.6(3.2-4.0)$ & $<0.0001$ \\
\hline PAC (pmol/L) & $926(601-1294)$ & $639(392-942)$ & $<0.0001$ \\
\hline PRA (ng/mL/h) & $0.20(0.11-0.22)$ & $0.20(0.11-0.32)$ & 0.859 \\
\hline $\mathrm{DRC}(\mathrm{ng} / \mathrm{L})$ & $2.5(1.7-3.0)$ & $3.0(2.0-5.0)$ & 0.022 \\
\hline ARR (pmol/L/ng/mL/h) & $5233(3110-7784)$ & $2958(1686-4470)$ & $<0.0001$ \\
\hline ARR (pmol/L/ng/L) & $413(244-797)$ & $251(160-519)$ & 0.008 \\
\hline Adenoma size on CT scan (mm) & $16(13-20)$ & $13(10-18)$ & 0.002 \\
\hline Radiological lesion & $82 \% \cup, 9 \% B, 9 \% N$ & $56 \% U, 16 \% B, 28 \% N$ & $<0.0001$ \\
\hline Basal LI & $20.54(10.17-41.43)$ & $3.71(1.65-11.54)$ & $<0.0001$ \\
\hline Post ACTH LI & $22.80(9.54-39.25)$ & $3.14(1.52-9.82)$ & $<0.0001$ \\
\hline Complete clinical cure at 12 months & $15 / 32(47 \%)$ & $9 / 50(18 \%)$ & 0.0049 \\
\hline $\begin{array}{l}\text { Complete biochemical cure at } 12 \text { months } \\
\text { (at the cut-off of } 6.15: A_{C L} / A_{p} \leq 6.15 \text { ) }\end{array}$ & $54 / 57(95 \%)$ & $10 / 20(50 \%)$ & $<0.0001$ \\
\hline
\end{tabular}

B, bilateral; DBP, diastolic blood pressure; DRC, direct renin concentration; N, normal; PRA, plasma renin activity; SBP, systolic blood pressure; $U$, unilateral.

ACTH LI > 10 were found to have lower basal contralateral aldosterone suppression and better surgical outcomes than those with LI between 4 and 9.9 (Supplementary Table 1). Individual values of the 17 patients who did not achieve biochemical cure after adrenalectomy are shown in Supplementary Table 2; although several cases clearly had low basal contralateral suppression, some patients with high LI and low contralateral suppression still failed to achieve biochemical cure.

\section{Discussion}

In this study, we confirmed in a larger cohort of 330 patients with PA from two different Canadian referral centers the conclusions of our initial study (14) that contralateral aldosterone suppression using the $A_{C L} / A_{P}$ ratio is relatively rare and usually incomplete in patients with lateralized source of excess aldosterone compared to what was previously reported using the $(\mathrm{A} / \mathrm{C})_{\mathrm{CL}} /(\mathrm{A} / \mathrm{C})_{\mathrm{P}}$ ratio $(8,9,10,11,12,13)$. Our results show that contralateral suppression is found in only $6 \%$ of our cohort of patients. In contrast, the traditional ratio of $(\mathrm{A} / \mathrm{C})_{\mathrm{CL}} /(\mathrm{A} / \mathrm{C})_{\mathrm{P}}$ is overestimating contralateral suppression at a cut-off of 1 (45\%), similar to what was found by others $(6,7,8,9,10$, 11). The median contralateral aldosterone level was found to be 2.3 times the concentration of aldosterone measured in the peripheral vein in patients with lateralized aldosterone secretion. The infrequent and incomplete contralateral suppression is probably secondary to the frequent presence of nodular zona glomerulosa hyperplasia including aldosterone-producing cell clusters (APCC) adjacent to the dominant nodule and most likely also in the zona glomerulosa of the contralateral adrenal gland (16). This incomplete contralateral suppression may also explain the very rare occurrence of hypoaldosteronism in the postoperative period, as reported in the literature (15) and also in our cohort (5.6\%). Unfortunately, during this retrospective study, no detailed pathology using immunohistochemistry to localize aldosterone synthetase expression was conducted to identify the presence of APCC and nodular zona glomerulosa hyperplasia in resected adrenals adjacent to dominant APA (22). However, in the Montreal cohort, using hematoxylin-eosin staining, pathological reports noted only one $(1.7 \%)$ case of zona glomerulosa atrophy adjacent to the APA; 24 (40\%) cases showed normal adjacent tissue and 35 (58.3\%) demonstrated a certain degree of nodular hyperplasia of the zona glomerulosa.

Several arguments led us to propose that $(\mathrm{A} / \mathrm{C})_{\mathrm{CL}} /$ $(\mathrm{A} / \mathrm{C})_{\mathrm{P}}$ ratio is inadequate to estimate contralateral suppression. A dominant APA should lead to suppression of contralateral aldosterone, but not of cortisol secretion regulated by a distinct secretory mechanism. Cortisolcorrected ratios correct for the different blood flows and dilution present in the left (by renal vein flow) compared to the right adrenal vein; however, there is no rationale to correct PAC by the cortisol in the peripheral vein. 
Contralateral suppression ratio should be based on basal samples to reflect the expected suppressed zona glomerulosa state rather than under pharmacological ACTH stimulation.

In previous studies, there were controversies in the predictive value of contralateral suppression for the postoperative cure, but most used cortisol-corrected ratios and they were often calculated after ACTH stimulation (8, $9,10,11,12,13)$. In the present study, we demonstrate that $\mathrm{A}_{\mathrm{CL}} / \mathrm{A}_{\mathrm{P}}$ at the cut-off of 2.15 is associated with postoperative clinical cure at 3 months and $\geq 12$ months, but not with the $(\mathrm{A} / \mathrm{C})_{\mathrm{CL}} /(\mathrm{A} / \mathrm{C})_{\mathrm{P}}$ ratio (Fig. 3). Both methods predicted the biochemical postoperative outcome at $>12$ months but with a high cut-off (6.15). ROC curve analysis was performed with data obtained only from patients undergoing unilateral adrenalectomy based on these cut-offs utilized in the two centers. This might be explained by the fact that biochemical cure is achieved in a very high proportion of patients after surgery, as a large proportion of aldosterone secreting cells is removed. It is also possible that remaining modest contralateral zona glomerulosa hyperplasia is clinically significant, but not biochemically distinct from the insensitive criteria of 'normal' ARR. The cut-offs determined by ROC curve are different from the one obtained in our previous study of 1.5 for global postoperative cure. This could be explained by current larger sample size and by the two different cut-

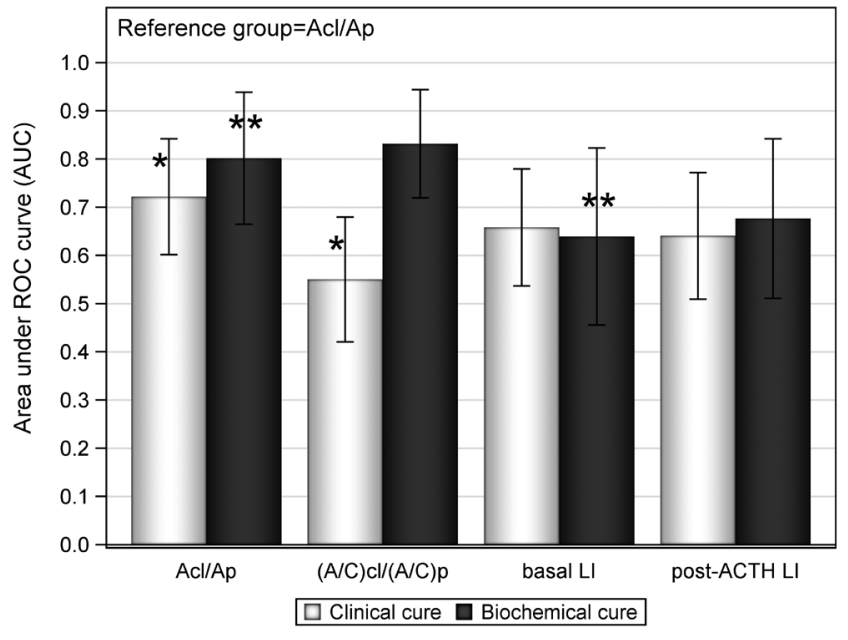

\section{Figure 3}

Comparison of the area under the ROC curve at 12 months for clinical and biochemical cures between the contralateral suppression $\left(A_{C L} / A_{p}\right.$ and $\left.(A / C)_{C L} /(A / C)_{P}\right)$ and the lateralization index (basal and post-ACTH). *Statistically different AUC compared to $A_{C L} / A_{p}$ for clinical outcome. **Statistically different $A \cup C$ compared to $A_{C L} / A_{P}$ for biochemical outcome. offs specific for clinical and the biochemical cures now based on the PASO consensus criteria (18).

We also found that the basal LI is a statistically less accurate parameter than $\mathrm{A}_{\mathrm{CL}} / \mathrm{A}_{\mathrm{P}}$ in predicting the biochemical cure at 12 months (Fig. 3). This is a surprising result as the basal contralateral suppression is incomplete in most cases of lateralized PA. It probably reflects that, although a LI above the cut-off of 2 basally and 4 post ACTH indicates a predominant aldosterone lateralized source, it does not demonstrate clearly that a unique APA is present on the same side as shown by more detailed IHC studies for CYP11B2 expression $(21,22)$. As expected, however, patients with higher LI $(>10)$ had lower basal aldosterone contralateral suppression and more favorable outcome following unilateral adrenalectomy (Supplementary Table 1).

The differences in severity of disease and surgical outcome between patients from the Montreal and Calgary cohorts are similar to those reported between various major centers in the PASO study and probably reflect differences in selection criteria to perform AVS (18). Our criteria for recommending unilateral adrenalectomy and for evaluation of postoperative outcomes are distinct but similar to those reported in several major centers taking care of PA in different countries (6). In the AVIS-2 multinational study, LI $>2$ was the most frequently used cut-off for basal sampling (7). The Calgary group identified that the more stringent Endocrine Society guidelines LI criteria ( $>4$ post ACTH) would have missed up to $40 \%$ of unilateral cases who benefited from surgery (23). The $83 \%$ of complete biochemical cure are also within the range found in the PASO international study (18). Many factors can explain why there is only a limited number of patients with complete postoperative cure: age, underlying essential hypertension, family history of hypertension, long-term vascular changes associated with long-standing primary aldosteronism and impaired renal function (24). However, we suggest that another factor is the frequent presence of contralateral zona glomerulosa hyperplasia, as measured by the basal contralateral suppression ratio.

When retrospectively reviewing the cases of this study, the recommended cut-offs for the lateralization in each center were not always respected for the decision of unilateral adrenalectomy. As shown in our and other studies, stimulation with ACTH helps improve the selectivity of AVS, but can lead to a discordance of lateralization between basal and post-ATCH values (14). In the current study, this happened in $22 \%$ of patients and most were lateralized basally but became bilateral post ACTH administration (in 19\% and in 3\% for the 
opposite situation). For those patients, it is more difficult to recommend the proper treatment. In Montreal, when discordance is present, in recent years, we rely mostly on basal ratios rather than post-ACTH ratios and we find it useful to jointly evaluate for contralateral aldosterone suppression to better estimate the chances of postoperative cure. If the patient clearly lateralizes basally and there is contralateral aldosterone suppression, we recommend surgery even if the LI is $<4$ post-ACTH stimulation. Inversely, if AVS clearly shows a bilateral source basally and that contralateral aldosterone suppression is absent, we favor medical treatment even if the $\mathrm{LI}$ is $>4$ post-ACTH. In earlier years from this study, as post-ACTH LI was our main surgical criteria, some patients who were diagnosed as having a bilateral source did not undergo surgery, but in retrospect had lateralized basal LI and contralateral suppression; these patients explain why rare patients diagnosed as being bilateral had basal contralateral suppression (Table 2). Individual review of operated cases which failed to achieve biochemical cure clearly indicate that no criteria of LI or contralateral suppression can accurately predict surgical success in all cases.

The strengths of this study are based on the validation of our previous results (14) with a larger number of PA patients studied in two centers using a similar bilateral simultaneous basal and post-ACTH stimulation protocol. This confirmed the rare and incomplete contralateral suppression in lateralized cases. The larger number of patients undergoing unilateral adrenalectomy in this combined study allowed to identify the relative values of contralateral suppression and LI in predicting surgical outcomes. Limitations result from the retrospective nature and the proportion of missing postoperative data at 12 months to assess the clinical and the biochemical outcomes. As in any multicenter study, various steroid assays utilized over time between centers may influence cut-offs for LI criteria, but as all analysis rely on ratios using same assays for each patient, we do not believe that this impacted our conclusions.

In summary, the basal $\mathrm{A}_{\mathrm{CL}} / \mathrm{A}_{\mathrm{P}}$ ratio should be utilized to evaluate contralateral suppression in patients with lateralized AVS to estimate the probable degree of zona glomerulosa hyperplasia in the adrenal contralateral to the dominant APA. A cut-off of 2.15 for basal $A_{C L} / A_{P}$ ratio significantly correlates with postoperative clinical success and helps identify the patients that will most benefit from unilateral adrenalectomy. It is a useful, but only modest, predictive parameter to use during AVS to predict the surgical outcome; this study indicates that it may be complementary to the classically used LI to select patients for whom unilateral adrenalectomy will be most beneficial. Clearly, contralateral aldosterone suppression is less prevalent than previously thought and reflects the frequent contralateral zona glomerulosa nodular hyperplasia which may contribute to the frequent persistence of milder degree of hypertension and may indicate the use of mineralocorticoid receptor antagonist if low-grade PA persists.

\section{Supplementary materials}

This is linked to the online version of the paper at https://doi.org/10.1530/ EJE-20-0254.

\section{Declaration of interest}

André Lacroix is on the editorial board of the European Journal of Endocrinology. André Lacroix was not involved in the review or editorial process for this paper, on which he is listed as an author. The other authors have nothing to disclose.

\section{Funding}

This research did not receive any specific grant from any funding agency in the public, commercial or not-for-profit sector.

\section{Acknowledgements}

The authors acknowledge the following colleagues for their contributions: nurses Shirley Ferguson, Sarah Haag, Lucretia Virlan and Emmanuel Byiringiro for coordination of adrenal vein sampling; Dr Mathieu Latour for pathological studies; Drs Marc Martin and Jean-Marie Boutin for hormone assays; and Alina Dyachenko for statistical analysis.

\section{References}

1 Monticone S, Burrello J, Tizzani D, Bertello C, Viola A, Buffolo F, Gabetti L, Mengozzi G, Williams TA, Rabbia F et al. Prevalence and clinical manifestations of primary aldosteronism encountered in primary care practice. Journal of the American College of Cardiology 201769 1811-1820. (https://doi.org/10.1016/J.JACC.2017.01.052)

2 Hannemann A \& Wallaschofski H. Prevalence of primary aldosteronism in patient's cohorts and in population-based studies - a review of the current literature. Hormone and Metabolic Research 201244 157-162. (https://doi.org/10.1055/s-0031-1295438)

3 Calhoun DA. Is there an unrecognized epidemic of primary aldosteronism? (Pro). Hypertension 200750 447-453; discussion 447. (https://doi.org/10.1161/HYPERTENSIONAHA.106.086116)

4 Monticone S, D'Ascenzo F, Moretti C, Williams TA, Veglio F, Gaita F $\&$ Mulatero P. Cardiovascular events and target organ damage in primary aldosteronism compared with essential hypertension: a systematic review and meta-analysis. Lancet: Diabetes and Endocrinology 20186 41-50. (https://doi.org/10.1016/S22138587(17)30319-4)

5 Funder JW, Carey RM, Mantero F, Murad MH, Reincke M, Shibata H, Stowasser M \& Young WF. The management of primary aldosteronism: case detection, diagnosis, and treatment: an endocrine society clinical practice guideline. Journal of Clinical Endocrinology and Metabolism 2016101 1889-1916. (https://doi. org/10.1210/jc.2015-4061) 
6 Rossi GP, Barisa M, Allolio B, Auchus RJ, Amar L, Cohen D, Degenhart C, Deinum J, Fisher E, Gordon R et al. The adrenal vein sampling International study (avis) for identifying the major subtypes of primary aldosteronism. Journal of Clinical Endocrinology and Metabolism 201297 1606-1614. (https://doi.org/10.1210/ jc.2011-2830)

7 Rossitto G, Amar L, Azizi M, Riester A, Reincke M, Degenhart C, Widimsky J, Mitsuhide N, Deinum J, Schultzekool L et al. Subtyping of primary aldosteronism in the AVIS-2 study: assessment of selectivity and lateralization. Journal of Clinical Endocrinology and Metabolism 2020105 dgz017. (https://doi.org/10.1210/clinem/ dgz017)

8 Kline GA, Chin A, So B, Harvey A \& Pasieka JL. Defining contralateral adrenal suppression in primary aldosteronism: implications for diagnosis and outcome. Clinical Endocrinology 201583 20-27. (https://doi.org/10.1111/cen.12669)

9 Wolley MJ, Gordon RD, Ahmed AH \& Stowasser M. Does contralateral suppression at adrenal venous sampling predict outcome following unilateral adrenalectomy for primary aldosteronism? A retrospective study. Journal of Clinical Endocrinology and Metabolism 2015100 1477-1484. (https://doi.org/10.1210/ jc.2014-3676)

10 Umakoshi H, Tanase-Nakao K, Wada N, Ichijo T, Sone M, Inagaki N, Katambi T, Kamemura K, Matsuda Y, Fujii Y et al. Importance of contralateral aldosterone suppression during adrenal vein sampling in the subtype evaluation of primary aldosteronism. Clinical Endocrinology 201583 462-467. (https://doi.org/10.1111/cen.12761)

11 Monticone S, Satoh F, Viola A, Fischer E, Vonend O, Bernini G, Lucatello B, Quinkler M, Ronconi V, Morimoto R et al. Aldosterone suppression on contralateral adrenal during adrenal vein sampling does not predict blood pressure response after adrenalectomy. Journal of Clinical Endocrinology and Metabolism 201499 4158-4166. (https:// doi.org/10.1210/jc.2014-2345)

12 Tagawa M, Ghosn M, Wachtel H, Fraker D, Townsend RR, Trerotola S $\&$ Cohen DL. Lateralization index but not contralateral suppression at adrenal vein sampling predicts improvement in blood pressure after adrenalectomy for primary aldosteronism. Journal of Human Hypertension 201731 444-449. (https://doi.org/10.1038/jhh.2016.92)

13 Fischer E, Degenhart C \& Reincke M. Improving adrenal venous sampling in primary aldosteronism. Expert Review of Endocrinology and Metabolism 20127 531-540. (https://doi.org/10.1586/eem.12.44)

14 El Ghorayeb N, Mazzuco TL, Bourdeau I, Mailhot JP, Zhu PS, Thérasse E \& Lacroix A. Basal and post-ACTH aldosterone and its ratios are useful during adrenal vein sampling in primary aldosteronism. Journal of Clinical Endocrinology and Metabolism 2016 101 1826-1835. (https://doi.org/10.1210/jc.2015-3915)

15 Fischer E, Hanslik G, Pallauf A, Degenhart C, Linsenmaier U, Beuschlein F, Bidlingmaier M, Mussack T, Ladurner R, Hallfeldt K et al. Prolonged zona glomerulosa insufficiency causing hyperkalemia in primary aldosteronism after adrenalectomy. Journal of Clinical Endocrinology and Metabolism 201297 3965-3973. (https://doi. org/10.1210/jc.2012-2234)

16 Boulkroun S, Samson-Couterie B, Golib Dzib JF, Lefebvre H, Louiset E, Amar L, Plouin PF, Lalli E, Jeunemaitre X, Benecke A et al. Adrenal cortex remodeling and functional zona glomerulosa hyperplasia in primary aldosteronism. Hypertension 201056 885-892. (https://doi.org/10.1161/HYPERTENSIONAHA.110.158543)

17 Fernandes-Rosa FL, Giscos-Duriez I, Amar L, Gomez-Sanchez CE, Meatchi T, Boulkroun S \& Zennaro MC. Different somatic mutations in multinodular adrenals with aldosterone producing adenoma. Hypertension 201666 1014-1022. (https://doi.org/10.1161/ HYPERTENSIONAHA.115.05993.Different)

18 Williams TA, Lenders JWM, Mulatero P, Burrello J, Rottenkolber M, Adolf C, Satoh F, Amar L, Quinkler M, Deinum J et al. Outcomes after adrenalectomy for unilateral primary aldosteronism: an international consensus on outcome measures and analysis of remission rates in an international cohort. Lancet. Diabetes and Endocrinology 20175 689-699. (https://doi.org/10.1016/S2213-8587(17)30135-3)

19 Hanley JA \& McNeil BJ. The meaning and use of the area under a receiver operating characteristic (ROC) curve. Radiology 1982143 29-36. (https://doi.org/10.1148/radiology.143.1.7063747)

20 DeLong ER, DeLong DM \& Clarke-Pearson DL. Comparing the areas under two or more correlated receiver operating characteristic curves: a nonparametric approach. Biometrics 198844 837-845. (https://doi. org $/ 10.2307 / 2531595)$

21 Zweig MH \& Campbell G. Receiver-operating characteristic (ROC) plots: a fundamental evaluation tool in clinical medicine. Clinical Chemistry 199339 561-577. (https://doi.org/10.1093/ clinchem/39.4.561)

22 Meyer LS, Wang X, Sušnik E, Burrello J, Burrello A, Castellano I, Eisenhofer G, Fallo F, Kline GA, Knösel T et al. Immunohistopathology and steroid profiles associated with biochemical outcomes after adrenalectomy for unilateral primary aldosteronism. Hypertension 201872 650-657. (https://doi. org/10.1161/HYPERTENSIONAHA.118.11465)

23 Kline G, Leung A, So B, Chin A, Harvey A \& Pasieka JL. Application of strict criteria in adrenal venous sampling increases the proportion of missed patients with unilateral disease who benefit from surgery for primary aldosteronism. Journal of Hypertension $2018 \mathbf{3 6}$ 1407-1413. (https://doi.org/10.1097/HJH.0000000000001693)

24 Rossi GP, Bolognesi M, Rizzoni D, Seccia TM, Piva A, Porteri E, Tiberio GAM, Giulini SM, Agabiti-Rosei E \& Pessina AC. Vascular remodeling and duration of hypertension predict outcome of adrenalectomy in primary aldosteronism patients. Hypertension 200851 1366-1371. (https://doi.org/10.1161/ HYPERTENSIONAHA.108.111369)

Received 20 March 2020

Revised version received 1 July 2020

Accepted 14 July 2020 